Original Article Journal of Epilepsy Research plSSN 2233-6249 / elSSN 2233-6257

Received May 24, 2021

Revised June 15, 2021

Accepted June 18, 2021

Corresponding author:

Dae-Won Seo, MD, PhD

Department of Neurology, Samsung

Medical Center, Sungkyunkwan University

School of Medicine, 81 Irwon-ro,

Gangnam-gu, Seoul 06351, Korea

Tel. $+82-2-3410-3599$

Fax. $+82-2-3410-0052$

E-mail; daewon@skku.edu

*Sunwoo JS and Jo HJ contributed to this work equally.

\section{Survey on Antiepileptic Drug Therapy in Patients with Drug Resistant Epilepsy}

Jun-Sang Sunwoo, MD, PhD ${ }^{1, *}$, Hyunjin Jo, MD ${ }^{2, *}$, Kyung Wook Kang, MD, PhD ${ }^{3}$, Keun Tae Kim, MD ${ }^{4}$, Daeyoung Kim, MD, PhD ${ }^{5}$, Dong Wook Kim, MD, PhD ${ }^{6}$, Min-Jee Kim, MD ${ }^{7}$, Saeyoon Kim, MD, PhD ${ }^{8}$, Woojun Kim, MD, PhD ${ }^{9}$, Hye-Jin Moon, MD ${ }^{10}$, Ha Ree Park, MD ${ }^{11}$, Jung-lck Byun, MD, PhD ${ }^{12}$, Jong-Geun Seo, MD, PhD ${ }^{13}$, Sung Chul Lim, MD, PhD ${ }^{14}$, Min Kyung Chu, MD, PhD ${ }^{15}$, Su-Hyun Han, MD ${ }^{16}$, Kyoung Jin Hwang, MD, PhD ${ }^{17}$, Dae-Won Seo, MD, PhD ${ }^{2}$ on behalf of the Drug Committee of Korean Epilepsy Society

${ }^{1}$ Department of Neurosurgery, Seoul National University Hospital, Seoul; ${ }^{2}$ Department of Neurology, Samsung Medical Center, Sungkyunkwan University School of Medicine, Seoul, ${ }^{3}$ Department of Neurology, Chonnam National University Hospital, Chonnam National University School of Medicine, Gwangju; ${ }^{4}$ Department of Neurology, Keimyung University School of Medicine, Daegu; ${ }^{5}$ Department of Neurology, Chungnam National University Hospital, Chungnam National University College of Medicine, Daejeon; ${ }^{6}$ Department of Neurology, Konkuk University School of Medicine, Seoul; ${ }^{7}$ Department of Pediatrics, Asan Medical Center Children's Hospital, University of Ulsan College of Medicine, Seoul; ${ }^{8}$ Department of Pediatrics, College of Medicine, Yeungnam University, Daegu; ${ }^{9}$ Department of Neurology, The Catholic University of Korea Seoul St. Mary's Hospital, Seoul; ${ }^{10}$ Department of Neurology, Soonchunhyang University Bucheon Hospital, Bucheon, ${ }^{11}$ Department of Neurology, Inje University Ilsan Paik Hospital, Inje University College of Medicine, Goyang, ${ }^{12}$ Department of Neurology, Kyung Hee University Hospital at Gangdong, Seoul, ${ }^{13}$ Department of Neurology, School of Medicine, Kyungpook National University, Daegu; ${ }^{14}$ Department of Neurology, The Catholic University of Korea St. Vincent's Hospital, Suwon; ${ }^{15}$ Department of Neurology, Severance Hospital, Seoul; ${ }^{16}$ Department of Neurology, Chung-Ang University Hospital, Chung-Ang University College of Medicine, Seoul, ${ }^{17}$ Department of Neurology, School of Medicine, Kyung Hee University, Seoul, Korea

Background and Purpose: Individualized anti-epileptic drug (AED) selection in patient with epilepsy is crucial. However, there is no unified opinion in treating patients with drug resistant epilepsy (DRE). This survey aimed to make a consolidate consensus with epileptologists' perspectives of the treatment for Korean DRE patients by survey responses.

Methods: The survey was conducted with Korean epilepsy experts who have experience prescribing AEDs via e-mail. Survey questionnaires consisted of six items regarding prescription patterns and practical questions in treating patients with DRE in Korea. The research period was from February 2021 to March 2021. Results: The survey response rate was $83.3 \%(90 / 108)$. Most $(77.8 \%)$ of the responders are neurologists. The proportion of patients whose seizures were not controlled by the second AED was $26.9 \%$. The proportion of patients who had taken five or more AEDs is $13.9 \%$, and those who are currently taking five or more AEDs are $7.3 \%$, of which $54.5 \%$ and $37.9 \%$ reported positive effects on additional AED, respectively. The majority $(91.1 \%)$ of respondents answered that the mechanism of action was the top priority factor when adding AED. Regarding data priority, responders considered that expert opinion should have the top priority, followed by clinical experiences, reimbursement guidelines and clinical evidence. Responders gave 64.9 points (range from 0 to 100) about overall satisfaction on reimbursement system of Health Insurance Review and Assessment Service for AED.

Conclusions: This study on AED therapy for DRE patients is the first nationwide trial in Korean epilepsy experts. In five drug failure, the top priorities on AED selection are mechanism of action and expert opinion. These findings might help to achieve consensus and recognize the insight on optimal therapy of AED in DRE. (2021;11:72-82)

Key words: Antiepileptic drugs, Drug resistant epilepsy, Epilepsy, Seizures 


\section{Introduction}

Epilepsy is a chronic disorder of the brain characterized by recurrent episodic attacks, epileptic seizures, and their somatic and psychiatric consequences. ${ }^{1}$ Epilepsy affects approximately 65 million people worldwide. ${ }^{2}$ The overall prevalence of treated epilepsy patients was 2.41/1,000, which is the period prevalence as of 2007. Gender-specific prevalence was higher in men than in women in all age groups. Its prevalence was the lowest in those in their 30s and 40s, and increased from the 50s age group in men, whereas the prevalence was nearly constant from the 40s age group in women. ${ }^{3}$

Anti-epileptic drug (AED) therapy is the mainstay of epilepsy treatment. Current treatment of AED follows a step approach that comprises initial mono-therapy and subsequent secondary mono- or initial add-on therapy, followed by the next drug trials using either mono-therapy or polytherapy. A variety of AEDs have been developed over the past few decades. Some are under development right now. Recently developed AEDs are categorized as the third generation of AEDs. Several compounds are under clinical investigation. They will be introduced to the market in near future. In Korea, about 20 AED are available currently. A few drugs are in process of obtaining approval from the Korea Food \& Drug Administration. Newly introduced AEDs show better safety profiles with new mechanisms of action. They have various mode of actions, including the following molecular mechanisms: 1) voltage-gated channel-related mechanism including blocking sodium or calcium channels or opening potassium channel; 2) neurotransmission-related mechanism such as modifying GABAergic or glutamatergic transmission; and 3) having specific molecular targets during synaptic vesicle transmission. ${ }^{4}$ A number of AEDs can work by more than one of the mechanism. However, our understanding about the entire prolife of AEDs' acting mechanisms are very limited. Moreover, although AEDs can suppress seizure, they cannot prevent or cure epilepsy. Despite there are a wider range of options of AEDs, about one-third of patients still suffer from drug resistant epilepsy (DRE) so far.

DRE patients face a significant trouble with potentially devastating neuropsychiatric dysfunction, psychosocial issues, a reduced quality of life, increased comorbidities, and even sudden unexpected death issues. ${ }^{5}$ Thus, early diagnosis and optimal therapy of patients with DRE are crucial. To achieve seizure freedom, clear clinical definition of DRE, knowledge of biomarkers, predictors, the pathophysiologic mechanism of DRE, and successful new therapy should be implicated. ${ }^{6-8}$ The underlying mechanism of DRE across a wide range of clinical settings and epilepsy syndrome remains unclear, although transporter hypothesis and target hypothesis have been suggested. ${ }^{4,9}$ An integrating concept incorporating a multifactorial process such as disease severity, genetic disorders, metabolic abnormalities, structural lesions, and network dysfunction with ongoing neural reorganization has been introduced. ${ }^{10,11}$ However, defining DRE is very difficult because the degree of intractability and the responsiveness to AED are highly variable according to the individual's state and disease course.

Despite many years of research, definitions of DRE still remain operational so far. An ad hoc task force of the International League Against Epilepsy (ILAE) defined drug resistance as "failure of adequate trials of two tolerated, appropriately chosen, and used anti-seizure medication schedules (whether as monotherapies or in combination) to achieve sustained seizure freedom" ${ }^{8}$ It appeared to have a high degree of interrater reliability. ${ }^{12,13}$ However, there are vague terms such as "failure", "adequate trials", and "sustained seizure freedom" in the ILAE definition of DRE. Moreover, the practical criterion "the two drug schedules" was formed in the era of the second generation of AEDs more than 20 years ago. The definition of DRE means that refractoriness can occur independent of the chosen AED. However, we are in the era of the third generation of AEDs with the capability of fully choosing an optimal treatment with about 20 AEDs. In addition, newly developed AEDs have evolutionary pharmacokinetic profiles, revolutionary acting mechanisms, and specific syndrome-targeting orphan drugs. These two drug criteria might be inappropriate to the current therapy. The operational concept would be in conflict with the current knowledge of underlying mechanism of DRE because diversely acting AEDs can affect many molecular targets at the same time. ${ }^{14}$

Epilepsy experts in real practice struggle with diagnosis and treatment of those patients in terms of patient's explanation, clinical application of treatment, determination of prognosis, a unified collection of patients for future research, and so on. The guidance for DRE patients has highlighted recent advances in patient-specific precision medicine. ${ }^{15-17}$ Rapidly developing method of neurogenetics and neuroimmunology, arterial intelligence, and big data technology can also be merged with AED thearpy. ${ }^{18,19}$ In the present choice of AED, disease-oriented concept in the past has been changed to a patient-oriented concept that is based on comprehensive multi-dimensional assessment of individual patients.

Therefore, the Drug Committee of Korean Epilepsy Society performed this study to understand the perception of Korean epilepsy 


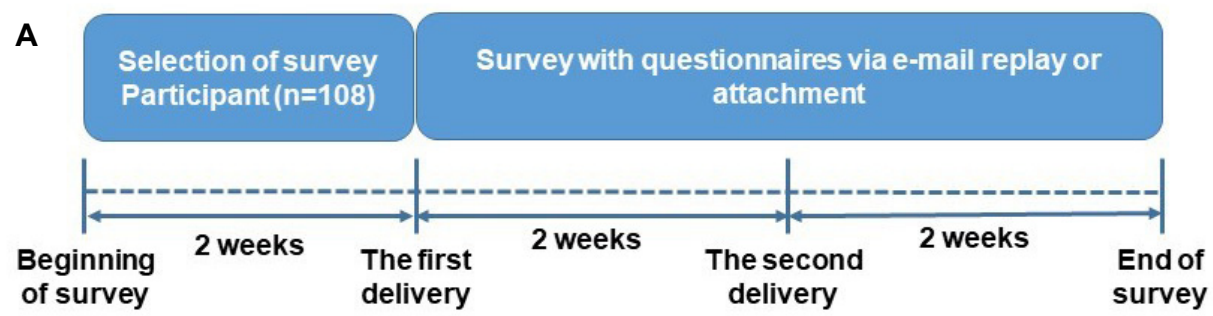

B

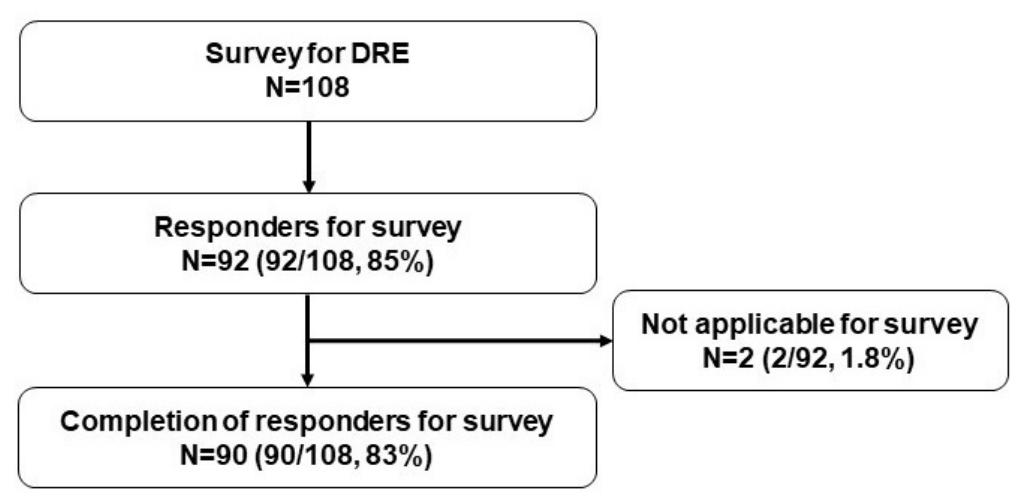

Figure 1. Study design. (A) Time schedule for the survey. (B) Responder disposition and key steps involved in the survey for anti-epileptic drug medication.

experts by analyzing approaches of AED selection for DRE patients in consideration of clinical experience along with the magnitude and location of the medical institution. Regarding treatment failure, the questions included both "two drug failure" and "five drug failure", reflecting recent common polytherapy in treating DRE. It will be beneficial to apply these results to real clinicians, which support them to achieve consensus in the treatment of DRE patients as well as to recognize the insight into DRE.

\section{Methods}

\section{Survey participants and methods}

The survey was conducted with 108 eligible experts (neurologists, neurosurgeons, and pediatricians) who actively participate in the treatment of patients with epilepsy. They were in the drug or epidemiology committees of Korean epilepsy society with experience in prescribing AEDs or at least 1-year clinical experience of epilepsy clinic practice. A total of 108 eligible participants received the survey e-mail for approximately 2 weeks. Responding e-mails were gathered within the first 2 weeks after receiving the first e-mail. Another 2 weeks after the second e-mail were allowed for participants who did not respond to the first email. The survey period was from
February 2021 to March 2021 (4-week period) (Fig. 1A). Overall, of 108 eligible participants for the survey, $92(85.2 \%)$ responded. However, responses from two (1.8\%) of these 92 were not applicable because they did not answer each item with the expression of their poor experience in DRE. Thus, responses from a total of 90 participants were analyzed (Fig. 1B). The survey was conducted nationwide. The factors such as gender (male/female), subspecialty (neurology, pediatrics, neurosurgery), clinical experience (less than 10 years, not less than 10 to less than 20 years, not less than 20 to less than 30 years, 30 years or more), hospital level (primary, secondary, tertiary, quaternary) ${ }_{1}^{20}$ and region (Seoul, Gyeonggi, Chungcheong, Jeolla, Jeju, Gyeonsang, and Gangwon) were considered as characteristics of survey respondents.

\section{Respondent demographics}

Of subjects of the survey, males accounted for $74.4 \%(n=67)$ and females for $25.6 \%$ ( $n=23)$. Regarding expertise, neurology $(77.8 \%$, $\mathrm{n}=70)$ occupied the highest percentage, followed by pediatrics $(18.9 \%, n=17)$ and neurosurgery $(3.3 \%, n=3)$. The median experience of the subjects was $15.0 \pm 8.22$ years (range, 1 year to 34 years). Subjects who had $\geq 10$ years but $<20$ years of experience accounted for $41.1 \%(n=37)$, those with $\geq 20$ years but $<30$ years of experience 
Table 1. Characteristics of survey responders

\begin{tabular}{lc}
\hline Item & Value \\
\hline Sex & $23(26.0)$ \\
Female & $67(74.0)$ \\
Male & \\
Expertise & $70(78.0)$ \\
Neurology & $3(3.0)$ \\
Neurosurgery & $17(19.0)$ \\
Pediatrics & $16.6 \pm 8.22$ \\
Duration of experience (years) & $7(7.8)$ \\
$\geq 30$ & $27(30.0)$ \\
$>30-20$ & $37(41.1)$ \\
$>20-10$ & $19(21.1)$ \\
$>10$ & $7(8.0)$ \\
Type of hospitals & $21(23.0)$ \\
Primary & $37(41.0)$ \\
Secondary & $25(28.0)$ \\
Tertiary & \\
Quaternary & $59(66.0)$ \\
Residential area & $12(13.0)$ \\
Seoul, Gyeonggi & $19(21.0)$ \\
Chungcheong, Jeolla, Jeju & \\
Gyeonsang, Gangwon & \\
\hline V & \\
\hline & \\
\hline &
\end{tabular}

Values are presented as mean \pm standard deviation or number (\%).

for $30 \%(n=27)$, those with $<10$ years of experience for $21.1 \%$ $(n=19)$, and those $\geq 30$ years of experience for $7.8 \%(n=7)$. Those who worked mainly in tertiary and quaternary hospitals accounted for the most $(68.9 \%, n=62)$, followed by those who worked in secondary hospitals $(23.3 \%, n=21)$ and primary hospitals $(8.9 \%, n=7)$. As for the working place locations, all provinces in Korea were included. Many of them were in Seoul and Gyeonggi $(65.6 \%, n=59)$ (Table 1).

\section{Survey questionnaire development}

The definition of DRE across survey items was developed referring to ILAE criteria. Overall survey questionnaires were composed of six items (Fig. 2). The first question asked about the percentage of treatment failures for the two drugs or more. In addition, sub-questions asked about the distribution of lack of effect, adverse event, adherence, and others for reasons judged to be uncontrolled causes. The second question was used to determine approximate rates of patients who failed five AEDs or more in the past, and the feasibility of efficacy on additional AEDs as a following question. In the third question, overall rates of patients treated with five AEDs or more currently and the possibility to be treated with additional AEDs were determined. The third question was developed through the number of AEDs with loss of seizure freedom rate ${ }^{14,21}$ and 2010 DRE severity proposal, ${ }^{22}$ as mentioned in the current evidence literature. The fourth item consisted of priorities with factors such as mechanism of action, hepatic enzyme inducer, pharmacokinetic interaction, adverse events, and others when adding AEDs to those who had control failures with five AEDs or more in the past. The fifth question was about the importance of factors considering additional AED among the options: expert opinions, meta-analysis, textbook, clinical trials, reimbursement guideline, and others. The fifth question asked to list those options in order depending on the degree of importance. The final question asked about satisfaction rate on overall AED reimbursement system of HIRA with the range of 100 points and 5 scales.

\section{Survey questionnaires}

The primary objective of this survey was to evaluate the prescription pattern of AED for DRE patients treated with multiple AEDs in real world practice. We created the survey questionnaires concerning previous numbers of AEDs, factors for the next AED choice, and feasibility to reduction in seizure frequency for each patient. The survey includes questionnaires: 1) the proportion of patients who failed two AED treatments, 2) the reason for failure to achieve seizure reduction, 3) rates of patients experienced from five AEDs or more prior (with previous prescription), 4) the feasibility to achieve seizure control when adding AEDs, 5) the proportion of patients who failed five AED treatments or more (currently prescribed), 6) the feasibility to seizure control for the patients who failed five AEDs or more, 7) the importance of factors considering additional AED, 8) priorities of evidence for further AED prescription on those who failed to five AEDs or more, 9) and 10) satisfaction with AED reimbursements from HIRA government body (Table 1).

\section{Analysis and statistics}

The survey conducted with a total of 108 eligible responses, and results were collected via e-mail or replies with attachment. Data analyses were conducted in a descriptive way. To assess detailed items, all questions were computed with response rates individually. Among ranking responses at question number 4 and 5 , rank 4 was a cut-off value. For sub-analysis, the Kruskal-Wallis test was used as a 


\section{Anti-Seizure Medication Survey}

항뇌전증약 설문(약물난치뇌전증 치료에 대해)

소 선생님의 클리닉 경험을 볼 때 적절한 답변 부탁드립니다.(노란색 칸에)

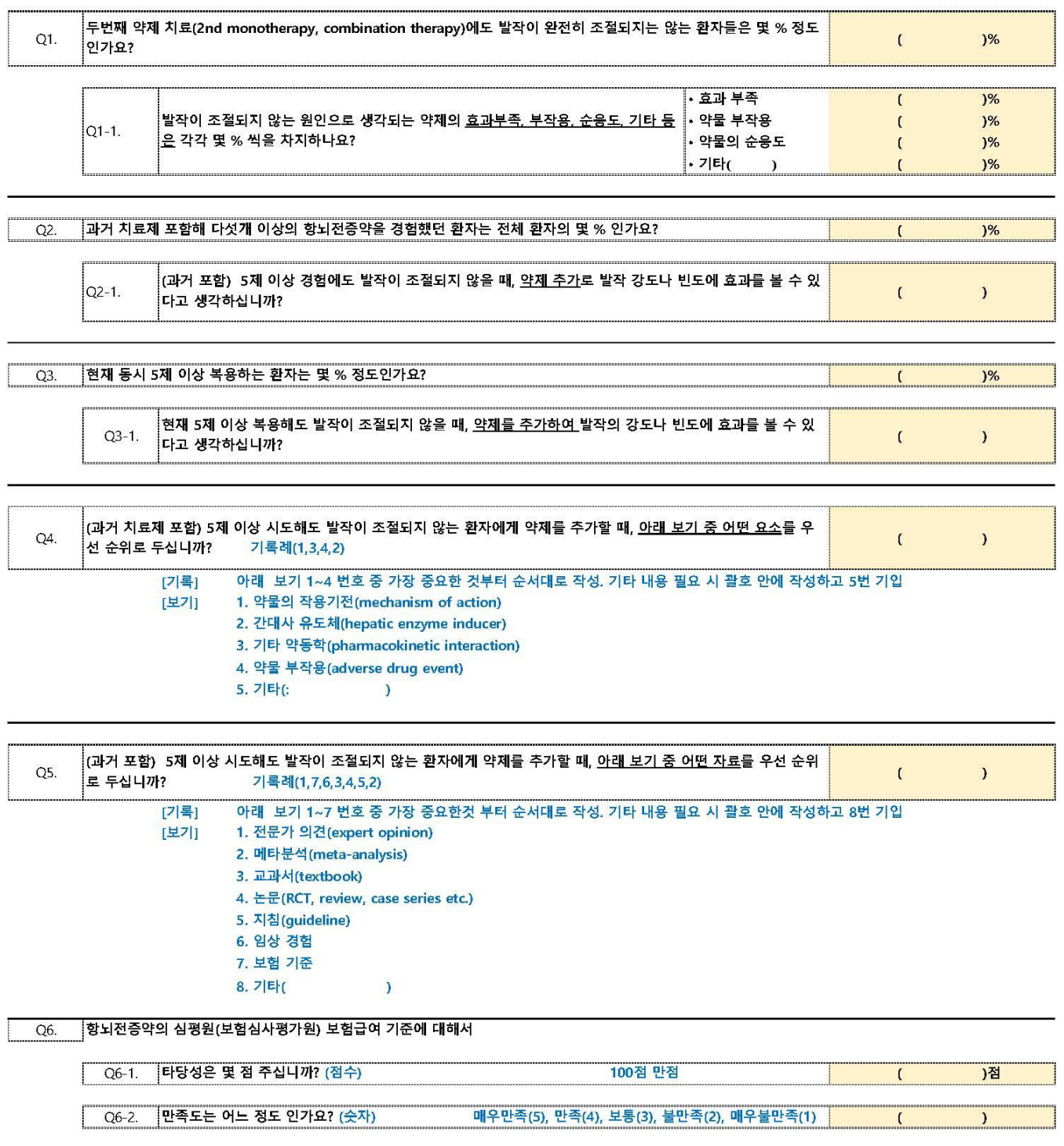

선생님의 고견에 진심으로 감사를 드립니다.

Figure 2. Survey form about anti-epileptic drug medication.

nonparametric statistical method. Overall data were divided by experience, region, hospital level, and expertise to confirm the statistical significance of each question. All analyses were performed using $R$ program (version 4.0.5; R Foundation for Statistical Computing, Vienna, Austria).

\section{Results}

The proportion of patients who failed two AEDs or more and reasons of failure to seizure control

The mean proportion of patients who failed in two AED treatments was $26.9 \pm 11.50 \%$ (range from $2 \%$ to $70 \%$ ). The causes of the fail- 
Table 2. Results of survey on items of drug failure and national insurance

\begin{tabular}{|c|c|}
\hline & Value \\
\hline Q1. The proportion of two drug failure (including secondary monotherapy and combination therapy) (\%) & $26.9 \pm 11.50$ \\
\hline \multicolumn{2}{|l|}{ Q1-1. Reasons of failure } \\
\hline Efficacy & $57.4 \%$ \\
\hline Adverse events & $20.4 \%$ \\
\hline Adherence & $18.6 \%$ \\
\hline Others & $3.6 \%$ \\
\hline Q2. The proportion of five drug failure (including past medications) (\%) & $13.3 \pm 9.48$ \\
\hline \multicolumn{2}{|l|}{ Q2-1. Expectation of efficacy in additional add-on therapy } \\
\hline Positive & $23(54.5)$ \\
\hline Negative & $20(45.5)$ \\
\hline Q3. The proportion of five drug failure in current medications (\%) & $7.3 \pm 5.30$ \\
\hline \multicolumn{2}{|l|}{ Q3-1. Expectation of efficacy in additional add-on therapy } \\
\hline Positive & $14(37.9)$ \\
\hline Negative & $29(62.1)$ \\
\hline \multicolumn{2}{|l|}{ Q4. Satisfaction on national medical insurance on anti-epileptic drug medications } \\
\hline Q4-1. Overall satisfaction score (on a scale from 1 to 100) & $64.9 \pm 16.91$ \\
\hline \multicolumn{2}{|l|}{ Q4-2. Proportion according to degree of satisfaction } \\
\hline Very satisfied & $19(1.1)$ \\
\hline Satisfied & $11(28.1)$ \\
\hline Neutral & $9(38.2)$ \\
\hline Dissatisfied & $4(23.6)$ \\
\hline Very dissatisfied & $0(9.0)$ \\
\hline
\end{tabular}

Values are presented as mean \pm standard deviation or number (\%) unless otherwise indicated.

ure included lack of efficacy (57.4\%), adverse event (20.4\%), adherence $(18.6 \%)$, and others (3.6\%) (Table 2 ). In sub-analysis groups, long clinical experienced epileptologists tended to report higher proportion of two drug failure than short clinical experienced ones, but not statistically significant. Regardless of expertise, hospital institution, clinical experience, or region, the main reason for treatment failure was 'lack of efficacy'. Among reasons of failure, neurology experts were likely to consider 'adverse event' and 'adherence' than the others even though it was not statistically significant.

\section{Rates of patients experiencing five AEDs prior or more (with previous prescriptions) and feasibility to seizure control when adding AEDs}

The mean proportion of patients experienced five AEDs or more, including previous medications, was $13.3 \pm 9.48 \%$ (range, $1 \%$ to $60 \%$ ). When asked about possible control of seizures in these patients by adding AED further, 48 epileptologists (54.5\%) responded that it was feasible (Table 2). Regarding intractable proportion of five drug failure, results based on the expertise showed that the pro- portion was $13.9 \%$ in neurologists, $12.4 \%$ in pediatricians, and $5.7 \%$ in neurosurgeon. As per clinical experience analysis, results are shown below: $<10$ years, $12.4 \% ; 10-20$ years, $12.4 \% ; 20-30$ years, $13.6 \%$; and $\geq 30$ years, $12.7 \%$. In a geographic analysis, the results of Seoul, Gyeonggi $(11.8 \%, n=56)$, Chungcheong, Honam, Jeju $(16.5 \%, n=12)$, Gyeonsang, and Gangwon $(17.1 \%, n=19)$ did not show significant difference. Regarding the question about the feasibility of seizure control with additional AEDs by expertise, those with neurology $(59.4 \%)$, pediatrics $(43.8 \%)$, and neurosurgery $(0.0 \%)$ expertise responded that it was feasible. Depending on the medical institution, the positive response was $28.6 \%$ in primary hospital, $57.1 \%$ in secondary hospital, $52.8 \%$ in tertiary hospital, and $62.5 \%$ in quaternary hospital.

\section{The proportion of patients failed five AED treatments or more (currently prescribed) and the feasibility to achieve seizure control when adding AEDs}

The mean proportion of patients currently taking five AEDs or 
more was $7.3 \pm 5.30 \%$ (Table 2). In sub-analyses based on expertise, the rate was $7.6 \%$ in neurologist, $6.9 \%$ in pediatrician, and $2.3 \%$ in neurosurgeon. The result of the question "could benefit from more additional AEDs" was 37.9\% in "yes" response (Table 2). As per clinical experience analysis, results are shown below: $<10$ years, $16.7 \%$; $10-20$ years, $51.4 \%$; $20-30$ years, $28 \%$; and $\geq 30$ years, $57.1 \%$. Depending on the medical institution, the positive answer was $0.0 \%$ in primary hospital, $28.6 \%$ in secondary hospital, $31.4 \%$ in tertiary hospital, and $64.0 \%$ in quaternary hospital. In the detailed results asking the possibility of efficacy with additional AEDs, neurology $(40.3 \%, n=67)$ and pediatrics $(35.3 \%, n=17)$ showed similar results while neurosurgery $(0.0 \%, n=3)$ had extremely low result.

\section{The importance of factors considering additional AEDs}

For patients failed to five AEDs or more, the following factors were considered as priorities: 1) mechanism of action, 2) adverse event, 3) other pharmacokinetics, and 4) liver metabolism derivatives in order $(n=79)$ (Table 3). Other factors included patient's underlying disease, bioavailability, protein binding, and knowledge of the latest papers on AEDs. Regardless of type of expertise, medical institution, clinical experience and region, mechanism of action was top considered option in 72 responders $(91.1 \%)$, followed by adverse event which was considered by six (7.6\%).

\section{The priorities of evidence for further AED prescription on those who failed to five AEDs}

Under the same conditions, when it came to considering adding more AEDs, expert opinion was considered as top priority by respondents, followed by clinical experience, reimbursement guidelines, and clinical evidences in order from given items including expert opinion, meta-analysis, textbook, clinical experience, reimbursement guidelines, clinical evidences, and others (Table 4). The expert opinion was shown as top priority by 23 responders $(31.5 \%)$, followed by clinical experience in $14(19.2 \%)$, guidelines in $13(17.8 \%)$, clinical evidences such as review article and case reports in 10 $(13.7 \%)$, and the others in $13(17.8 \%)$. In the detailed analysis, the best considered item was "expert opinion" in those with neurology

Table 3. Results of survey on selection priority of antiepileptic drug medications in refractory patients (survey question number Q4)

\begin{tabular}{lcccc}
\hline \multirow{2}{*}{ Item } & \multicolumn{3}{c}{ Ranking } \\
\cline { 2 - 5 } & 1st & 2nd & 3rd & 4th \\
\hline Mechanism of action & $72(91.1)$ & $3(3.8)$ & $1(1.3)$ & $3(3.8)$ \\
Hepatic enzyme inducer & $0(0.0)$ & $10(12.7)$ & $28(35.4)$ & $39(49.4)$ \\
Other pharmacokinetic event & $0(0.0)$ & $20(25.3)$ & $33(41.8)$ & $25(31.6)$ \\
Adverse drug event & $6(7.6)$ & $46(58.2)$ & $17(21.5)$ & $10(12.7)$ \\
Others & $1(1.3)$ & $0(0.0)$ & $0(0.0)$ & $2(2.5)$ \\
Total & 79 & 79 & 79 & 79 \\
\hline
\end{tabular}

Values are presented as number (\%).

Table 4. Results of survey on referential resources (survey question number Q5)

\begin{tabular}{lcccc}
\hline \multirow{2}{*}{ Item } & \multicolumn{3}{c}{ Ranking } \\
\cline { 2 - 5 } & 1st & 2nd & 3rd & 4th \\
\hline Expert opinion & $23(31.5)$ & $15(20.5)$ & $10(13.7)$ & $11(15.1)$ \\
Meta-analysis & $4(5.5)$ & $7(9.6)$ & $13(17.8)$ & $22(30.1)$ \\
Textbook & $9(12.3)$ & $7(9.6)$ & $9(12.3)$ & $9(12.3)$ \\
Review, case series & $10(13.7)$ & $14(19.2)$ & $13(17.8)$ & $12(16.4)$ \\
Guidelines & $13(17.8)$ & $12(16.4)$ & $12(16.4)$ & $7(9.6)$ \\
Clinical experience & $14(19.2)$ & $15(20.5)$ & $12(16.4)$ & $7(9.6)$ \\
Medical insurance & $0(0.0)$ & $3(4.1)$ & $3(4.1)$ & $5(6.8)$ \\
Others & $0(0.0)$ & $0(0.0)$ & $1(1.4)$ & $0(0.0)$ \\
Total & 73 & 73 & 73 & 73 \\
\hline
\end{tabular}

Values are presented as number (\%). 
and pediatrics expertise, while "clinical evidences and clinical experiences" was chosen for those with expertise in neurosurgery. Expert opinion was also reported as the top priority according to responder's clinical experiences and regions.

\section{Satisfaction with AED reimbursements system from HIRA government body}

Overall average points for satisfaction on HIIRA reimbursement system for AED as $64.9 \pm 16.91$ points (range, 0 to 100 ) with very satisfied in one responder (1.1\%), satisfied in $25(27.8 \%)$, neutral in 34 $(37.8 \%)$, dissatisfied in $22(24.4 \%)$, and very dissatisfied in eight (8.9\%) in detail (Table 2).

\section{Discussion}

This survey targeted epilepsy experts prescribing AEDs to establish a comprehensive opinion on the prescribing status of epilepsy patients, especially DRE patients and factors to consider when performing AED selection. It was the first trial conducted with 108 experts in epilepsy nationwide. These experts had a great deal of experience in prescribing AED with clinical experiences in epilepsy clinic for at least 1 year. The place of participants consisted of all provinces in Korea. The e-mail survey was sent to responders to complete the survey file as attachment or reply with answers. It was conducted from February 2021 to March 2021. The survey participants showed a high response rate of $83 \%$ during a short period of 4 weeks.

Drug resistance in epilepsy is common. In AED treatment of newly diagnosed epilepsy patient, the seizure free rate of initial monotherapy is nearly about $60 \%$. The remaining patients are considered to become potentially DRE because the further seizure free rate in additional changed or combined AED therapy is less than $10 \%$. Historically comparative polytherapy trials have found that $11 \%$ to $35 \%$ of patients with partial seizures become seizure free, and additional $12 \%$ to $29 \%$ of patients have more than $50 \%$ seizure reduction. ${ }^{22-25}$ A review of 35 seizure studies showed that the pooled prevalence proportion of DRE was $30 \%{ }^{26}$ In the present study, failure rate of two drugs was about $26.9 \pm 11.50 \%$, in line with approximate rate of the previous data following the ILAE criterion for DRE. ${ }^{26}$ In the present study, the proportion of patients who failed five AEDs or more including history was $13.3 \pm 9.48 \%$. There was a subsequent question on whether additional AED treatment could help seizure control even in the failed cases with five failed AEDs or more prior. About $55 \%$ of responders said that the treatment would be effective if adding another AED to them. This reveals that there is still room for new AED trial even in DRE with five previous failed drugs or more. In sub-analysis, it was confirmed that $59.4 \%$ of neurologist and $57.1 \%$ of those with clinical experience $\geq 30$ years responded hopefully, indicating that neurology subspecialty and longer clinical experience tended to answer positively. Korean National Evidence-based Healthcare Collaborating Agency has reported that the percentage of AED 1 year before epilepsy surgery is $20.3 \%$ for those having $\leq 2$ drugs, $28.3 \%$ for those having three drugs, $26.0 \%$ for those having four drugs, and $25.4 \%$ for those having $\geq 5$ drugs (Chung CK, Lee JA; https://www.medric.or.kr; December 2015). In this Korean report, analysis of 1,443 refractory epilepsy patients who had undergone epilepsy surgery showed nearby two times higher percentage of patients on five drugs or more.

In the present study, the percentage of patients taking five failed AEDs or more currently at the same time was $7.3 \pm 5.30 \%$. The rate of responding that the treatment would be effective when another AED was added was $37.9 \%$ (62.1\% of ' $N o$ '). Considering positive thinking in more than one third of responders, an additional drug trial would be possible even for patients with five concurrent failed drugs or more. In sub-analysis, the majority (57.1\%) of those with $\geq 30$ years of clinical experience answered that it would be effective, while only $16.7 \%$ of those with $<10$ years of clinical experience mentioned that it would be effective. Depending on the medical institution and clinical experience, $100 \%$ of those in the primary hospital reported that it would not be effective, whereas $64 \%$ of them from quaternary hospital responded positively. Considering above results, a new drug trial in DRE could be much influenced by service level of the hospital and the degree of clinical experience.

In the present study, the reason for intractability in the patients with two failed AEDs medications was the lack of effectiveness in majority of respondents (57.4\%). Next to ineffectiveness, adverse events (20.4\%) and non-adherence (18.6\%) were common causes of intractability. The most common factor that leads to AED failure for seizure control in patients with DRE was lack of effectiveness. This suggests that even though there are currently 18 kinds of AEDs on the market in Korea, there are still unmet needs for new AEDs in real practice. Moreover, if drugs with new act mechanisms are developed, they might be able to achieve better efficacy in patients despite their previous exposure to various AEDs. Combination therapy with new AED having newly acting mechanism is likely to become popular because it is difficult to alleviate the number of seizures with currently existing AEDs. Thus, conventional two drug criteria for DRE cannot be 
practical. Studies in developing countries have shown non-adherence to anti-seizure medications occurs in $48.1-65.4 \% .{ }^{27,28}$ Common causes of non-adherence are forgetfulness, the inability to obtain medication, and medication belief. Socioeconomic factors are significantly related to adherence. ${ }^{28}$ In Korea, the national health insurance system is established well enough to lower the adherence issue. However, $20 \%$ of the DRE patients still had a drug adherence problem. Gollwitzer et al. ${ }^{29}$ have shown that good adherence is higher in patients with new than old, branded than generic AED, and once than two or more daily dosages. Thus, detailed counselling, selection of AED and dose regimen can be needed in every day practice to reduce non-adherence as a cause of intractability.

In the present study, when adding drugs to patients whose seizures were not controlled despite the use of five AEDs in the past, "mechanism of action" was the first priority, followed by "adverse event", "pharmacokinetics", and "hepatic metabolism". The percentage of selecting "mechanism of action" was absolutely high (91.1\%). According to expertise, medical institution, clinical experience, and region, results have no significant changes. To make polytherapy efficacious, the combination should focus on synergistic effects with safety and without toxicity. These results of this survey confirmed that experts' AED selection was identical to that shown in several well-known references, textbooks, and guidelines. ${ }^{30-33}$

As in the epilepsy textbook, tailored AED selection for the optimal treatment is based on the individual condition of each patient as well as traditional epilepsy syndrome or seizure type. The impact of seizure can be different depending on individual patient. Thus, the different impacts may induce different disease/therapy-associated alterations that can affect intrinsic severity as well as seizure frequency. The disease/therapy-associated alterations include molecular, cellular, and network changes and disease modifying effects (anti-inflammation, AED metabolism or distribution, change of efflux transport P-glycoprotein). ${ }^{34}$ In the context of DRE, putative drug-resistance mechanism and various factors including genetic factors should be considered according to the state of patients, because those factors are closely associated with seizure frequency of each patient. Add-on strategies in DRE might be considered, including the development of novel approaches to help patients to overcome DRE. Our survey results reflect recent concepts that mechanistic polytherapy of AED continues to be common practice, especially in institutionalized epilepsy patients. In addition, Korean experts positively think that new AED could be tried for patients who have failed five or more AEDs to increase the efficacy of polytherapy.
However, the necessity of AED polytherapy is not needed for all patients. Especially for the elderly, women of child-bearing potential, and children, AED overtreatment should be avoided since they are vulnerable to undesirable combinations of AED and non-AED polytherapy and subsequent adverse effects posing significant risks. ${ }^{35-37}$ In polytherapy, fatigue, dizziness, cognitive problems, memory complaints, depression, suicidal attempt, and sudden unexplained death in epilepsy patients (SUDEP) can be increased due to pharmacokinetic interactions, dose-related neurotoxic effects, pharmacodynamic reactions, additive or synergistic drug related toxicities, and teratogenicity. ${ }^{36-40}$ Polytherapy may be less tolerable than monotherapy because of a higher total drug load. ${ }^{41,42}$ One study suggested that the appropriate indications for AED polytherapy can include transitional polytherapy during titration of a new adjunctive AED toward monotherapy or long-term maintenance AED polytherapy in medically refractory epilepsy. ${ }^{43}$ Add-on therapy can also be tried for safety referring to the defined daily dose (DDD) values of the AEDs assigned by the World Health Organization. It has been reported that total daily dose is not controlled in most polytherapy studies. ${ }^{42,44}$ The prescribed daily dose to DDD ratio in monotherapy or polytherapy should be below 2 to reduce the likelihood of neurological side effects. In the present study, adverse event was the second priority following mechanism of action. It would be necessary to select an AED with improved adverse event. An optimal mechanism of action should also be given priority considering individual state of the patient. In this survey, next top priority was drug-drug interaction, which was derived from pharmacokinetic characteristics between AEDs. Experts preferred the evolutionary pharmacokinetic AED, lower hepatic metabolism (CYP450) regarding other medicine's interaction and special population in epilepsy. In the present survey, Korean epileptologists referred to expert opinion, clinical experience, clinical trials, and guidelines in order when additional AED is added to patients. As shown the result, experts were likely to prefer cumulative expert opinion rather than others since there was no unified opinion on personalized epilepsy patients.

Currently, 30 kinds of AEDs have been developed for decades. In Korea, 18 kinds of AEDs could be reimbursed. For epilepsy patients, customized precision medicine is essential based on individual seizure types and drug-related factors. From an epilepsy pathology point of view, it is a priority to select an AED with a suitable mechanism of action tailored to the patient in near future. In the case of recently developed third-generation drugs, brivaracetam (SV2A antagonist) and eslicarbazepine (persistent sodium channel blocker) have been de- 
veloped and they show advanced mode of actions. AEDs having new mechanisms of action are also under development. They will be introduced in the near future. They are expected to contribute to personalized medicine for each patient based on clinical perspective.

In summary, we investigated how experts would approach, diagnose, and prescribe appropriate drugs to patients with refractory epilepsy in Korea. Medical staffs with a lot of clinical experience have positive mind about the treatment of most DRE. Results confirmed that DRE could be approached with a method centering on the mechanism of action of a drug. If the standards for DRE patients were changed from two AEDs to not less than five including the past, not less than five drugs currently, there was a tendency of drug failure rate to decrease from $26.9 \%$ to $13.3 \%$ and $7.3 \%$, although there was no significant difference according to the characteristics of the respondents. In addition, many respondents affirmed that adding AEDs would have a positive effect even if patients had treatment failure with five AEDs. The mechanism of action of the AED as a factor for additional selection was considered first. Most epileptologists refer to the opinions of experts first. However, their satisfaction with the reimbursement system for the use of AEDs in the medical field seems to be limited to active treatment. The results of this survey are expected to contribute to the therapeutic significance, prognosis and future treatment considerations of Korean DRE patients in real clinical practice.

\section{Conflict of Interest}

The authors declare that they have no conflicts of interest.

\section{References}

1. Beghi E, Giussani G. Aging and the epidemiology of epilepsy. Neuroepidemiology 2018;51:216-23.

2. Thurman DJ, Beghi E, Begley CE, et al. Standards for epidemiologic studies and surveillance of epilepsy. Epilepsia 2011;52 Suppl 7:2-26.

3. Lee SY, Jung KY, Lee IK, et al. Prevalence of treated epilepsy in Korea based on national health insurance data. J Korean Med Sci 2012;27: 285-90.

4. Rogawski MA, Johnson MR. Intrinsic severity as a determinant of antiepileptic drug refractoriness. Epilepsy Curr 2008:8:127-30.

5. Janmohamed M, Brodie MJ, Kwan P. Pharmacoresistance - epidemiology, mechanisms, and impact on epilepsy treatment. Neuropharmacology 2020;168:107790.

6. Jarosiewicz B, Morrell M. The RNS system: brain-responsive neurostimulation for the treatment of epilepsy. Expert Rev Med Devices
20201;18:129-138.

7. Walker $L E$, Janigro $D$, Heinemann $U$, Riikonen $R$, Bernard C, Patel M. WONOEP appraisal: molecular and cellular biomarkers for epilepsy. Epilepsia 2016;57:1354-62.

8. Kwan P, Brodie MJ. Definition of refractory epilepsy: defining the indefinable? Lancet Neurol 2010;9:27-9.

9. Löscher W, Sills GJ. Drug resistance in epilepsy: why is a simple explanation not enough? Epilepsia 2007:48:2370-2.

10. Pohlmann-Eden B, Weaver DF. The puzzle(s) of pharmacoresistant epilepsy. Epilepsia 2013;54 Suppl 2:1-4.

11. Schmidt $D$, Löscher W. New developments in antiepileptic drug resistance: an integrative view. Epilepsy Curr 2009;9:47-52.

12. Kwan P, Schachter SC, Brodie MJ. Drug-resistant epilepsy. N Eng/ J Med 2011;365:919-26.

13. Hao XT, Wong IS, Kwan P. Interrater reliability of the international consensus definition of drug-resistant epilepsy: a pilot study. Epilepsy Behav 2011;22:388-90.

14. Löscher W, Potschka H, Sisodiya SM, Vezzani A. Drug resistance in epilepsy: clinical impact, potential mechanisms, and new innovative treatment options. Pharmacol Rev 2020;72:606-38.

15. Nabbout $R$, Kuchenbuch $M$. Impact of predictive, preventive and precision medicine strategies in epilepsy. Nat Rev Neurol 2020;16:674-88.

16. Li Y, Zhang S, Snyder MP, Meador KJ. Precision medicine in women with epilepsy: the challenge, systematic review, and future direction. Epilepsy Behav 2021;118:107928.

17. Chen Z, Rollo B, Antonic-Baker A, et al. New era of personalised epilepsy management. BMJ 2020;371:m3658.

18. Jang Y, Kim DW, Yang Kl, et al. Clinical approach to autoimmune epilepsy. J Clin Neurol 2020;16:519-29.

19. An S, Kang C, Lee HW. Artificial intelligence and computational approaches for epilepsy. J Epilepsy Res 2020;10:8-17.

20. Labiner DM, Bagic Al, Herman ST, et al. Essential services, personnel, and facilities in specialized epilepsy centers--revised 2010 guidelines. Epilepsia 2010;51:2322-33.

21. Johannessen Landmark C, Patsalos PN. Drug interactions involving the new second- and third-generation antiepileptic drugs. Expert Rev Neurother 2010;10:119-40.

22. Lorenzo NY, Bromfield EB, Theodore WH. Carbamazepine and phenytoin: combination versus single drug therapy. Eur J Neurol 1995;2:101-5.

23. Mattson RH, Cramer JA, Collins JF, et al. Comparison of carbamazepine, phenobarbital, phenytoin, and primidone in partial and secondarily generalized tonic-clonic seizures. N Eng/ J Med 1985;313:145-51.

24. Dean JC, Penry JK. Valproate monotherapy in 30 patients with partial seizures. Epilepsia 1988;29:140-4.

25. Tanganelli P, Regesta G. Vigabatrin vs. carbamazepine monotherapy in newly diagnosed focal epilepsy: a randomized response conditional cross-over study. Epilepsy Res 1996;25:257-62.

26. Kalilani L, Sun X, Pelgrims B, Noack-Rink M, Villanueva V. The epidemiology of drug-resistant epilepsy: a systematic review and meta-analysis. 
Epilepsia 2018;59:2179-93.

27. Liu J, Liu Z, Ding H, Yang X. Adherence to treatment and influencing factors in a sample of Chinese epilepsy patients. Epileptic Disord 2013; 15:289-94.

28. Niriayo YL, Mamo A, Gidey K, Demoz GT. edication belief and adherence among patients with epilepsy. Behav Neurol 2019;2019:2806341.

29. Gollwitzer S, Kostev K, Hagge M, Lang J, Graf W, Hamer HM. Nonadherence to antiepileptic drugs in Germany: a retrospective, population-based study. Neurology 2016;87:466-72.

30. Verrotti A, Tambucci $R$, Di Francesco $L$, et al. The role of polytherapy in the management of epilepsy: suggestions for rational antiepileptic drug selection. Expert Rev Neurother 2020;20:167-73.

31. Brodie MJ, Sills GJ. Combining antiepileptic drugs--rational polytherapy? Seizure 2011;20:369-75.

32. French JA, Faught E. Rational polytherapy. Epilepsia 2009;50 Suppl 8:63-8.

33. Giussani $G$, Beghi E. Does mechanism of drug action matter to inform rational polytherapy in epilepsy? CNS Neurol Disord Drug Targets 2013; 12:426-35.

34. Potschka H. Animal and human data: where are our concepts for drug-resistant epilepsy going? Epilepsia 2013;54 Suppl 2:29-32.

35. Crawford P. Epilepsy and pregnancy. Seizure 2002;11 Suppl A:212-9.
36. Oguni M, Osawa M. Epilepsy and pregnancy. Epilepsia 2004;45 Suppl 8:37-41.

37. Pennell PB. Antiepileptic drug pharmacokinetics during pregnancy and lactation. Neurology 2003;61(6 Suppl 2):S35-42.

38. Arroyo $S$, de la Morena A. Life-threatening adverse events of antiepileptic drugs. Epilepsy Res 2001;47:155-74.

39. Schmidt D. Strategies to prevent overtreatment with antiepileptic drugs in patients with epilepsy. Epilepsy Res 2002;52:61-9.

40. Schmidt D, Elger C, Holmes GL. Pharmacological overtreatment in epilepsy: mechanisms and management. Epilepsy Res 2002;52:3-14.

41. Deckers $C L$, Hekster YA, Keyser A, Meinardi H, Renier WO. Reappraisal of polytherapy in epilepsy: a critical review of drug load and adverse effects. Epilepsia 1997;38:570-5.

42. Deckers CL, Czuczwar SJ, Hekster YA, et al. Selection of antiepileptic drug polytherapy based on mechanisms of action: the evidence reviewed. Epilepsia 2000;41:1364-74.

43. St Louis EK. Minimizing AED adverse effects: improving quality of life in the interictal state in epilepsy care. Curr Neuropharmacol 2009;7: 106-14.

44. Deckers CL. Overtreatment in adults with epilepsy. Epilepsy Res 2002; 52:43-52. 